\title{
Impact of house dust mite-driven asthma on children's school performance and activity
}

\author{
Catalina Gómez ${ }^{1}$. Judit Barrena ${ }^{2}$. Vanesa García-Paz ${ }^{3}$. Ana M. Plaza ${ }^{4}$. Paula Crespo ${ }^{5}$ - José A. Bejarano ${ }^{5}$. \\ Ana B. Rodríguez ${ }^{5} \cdot$ Laia Ferré $^{1} \cdot$ Lidia Farrarons $^{1} \cdot$ Marta Viñas $^{2} \cdot$ Carla Torán-Barona $^{6} \cdot$ Andrea Pereiro $^{6}$. \\ José L. Justicia ${ }^{6}$. Santiago Nevot ${ }^{1}$
}

Received: 29 July 2021 / Revised: 31 October 2021 / Accepted: 9 December 2021 / Published online: 21 December 2021

(C) The Author(s) 2021

\begin{abstract}
Evidence regarding asthma's impact on children's daily lives is limited. This prospective and cross-sectional, observational, multicenter study assessed school/work and activity impairment in children and adolescents with allergic asthma and their caregivers and allergen immunotherapy (AIT) effects. Included patients were schooled children and adolescents (5 to 17 years) with allergic asthma due to house dust mites (HDM). Impairment of school/work (i.e., absenteeism and presenteeism) and activity was measured in patients and their caregivers using the Work Productivity Impairment Questionnaire plus Classroom Impairment Questions: Allergy Specific (WPAI + CIQ:AS). HDM allergic patients with school impairment received subcutaneous AIT with a MicroCrystalline Tyrosine-associated allergoid. WPAI + CIQ:AS and effectiveness variables were compared between baseline and 1-year post-AIT. Of the 113 patients included, $59(52.2 \%)$ and 51 (45.1\%) showed school and activity impairment, respectively, missing a mean (SD) of 37.6 (24.4) \% and 42.6 (25.6) \% of school and activity time, respectively. Twenty-six (23\%) caregivers reported activity impairment and, of the $79(69.9 \%)$ employed, 30 (38\%) reported work impairment. Of the 65 patients with school/activities impairment, 41 (63.1\%) received AIT, of which 21 (51.2\%) completed 1 year of treatment. Effectiveness variables and WPAI + CIQ:AS significantly improved: Mean (SD) school impairment decreased from $39.7(26.7)$ to 2.1 (7.1) \% ( $p<0.001)$ and activity impairment from $46.2(34.6)$ to $1.4(3.6) \%(p<0.001)$.

Conclusion: Allergic asthma due to HDMs results in school/work and activity impairment in children and adolescents and their caregivers. One year of AIT provided clinical benefits and reduced school and activity impairment.

\section{What is Known:}

- Allergic asthma impairs children's school performance and daily activities.

- Allergen immunotherapy modifies allergic disease course and ameliorates its symptoms.

What is New:

- Asthma symptoms due to allergy to house dust mites impair children's school attendance and productivity and daily activity and their caregivers' work performance and daily lives.

- Allergen immunotherapy with a house dust mite MicroCrystalline Tyrosine (MCT)-associated allergoid seems to provide clinical benefits, associated with decreased school and activity impairment, supporting it as an effective treatment option.
\end{abstract}

Keywords School impairment · Absenteeism · Work impairment · Subcutaneous allergen immunotherapy · Microcrystalline tyrosine $\cdot$ Allergic asthma

Communicated by Nicole Ritz

Carla Torán-Barona

Carla.Toran@allergytherapeutics.com

1 Althaia Xarxa Assistencial I Universitària de Manresa, Manresa, Spain

2 Consorci Sanitari de Terrassa, Terrassa, Spain
3 Hospital Quirón, Coruña, Spain

Hospital Sant Joan de Déu, Esplugues de Llobregat, Spain

5 Hospital Virgen del Rocío, Sevilla, Spain

6 Allergy Therapeutics Ibérica, Avda. Barcelona, 115, 08970 Sant Joan Despí, Barcelona, Spain 


\begin{tabular}{ll}
\multicolumn{2}{l}{ Abbreviations } \\
AIT & Allergen immunotherapy \\
GEMA & "Guía Española del Manejo del Asma" \\
GINA & Global Initiative for Asthma \\
HDMs & House dust mites \\
LABAs & Long-acting beta-2 agonists \\
LTRAs & Leukotriene receptor antagonists \\
MCT & MicroCrystalline Tyrosine \\
QoL & Quality of life \\
SABAs & Short-acting beta-2 agonists \\
SD & Standard deviation \\
WPAI+CIQ & AS Work Productivity Impairment \\
& Questionnaire plus Classroom Impairment \\
& Questions: Allergy Specific
\end{tabular}

\section{Introduction}

Allergic diseases, including asthma, rhinitis, and atopic dermatitis, impair multiple aspects of patients' daily functioning, causing a serious burden and impaired quality of life [1]. In children, asthma is one of the most common chronic diseases, with an estimated global symptom prevalence of 11.6 to $13.7 \%$, and is frequently associated with allergic rhinitis [2]. Among the most frequent allergens associated with allergic asthma, those from house dust mites (HDMs) are common in indoor environments (i.e., at home) and rank second after pollens, constituting the main cause of perennial allergic asthma in Spain [3, 4]. Despite the increased awareness of asthma in children and availability of effective treatment, asthma control is still suboptimal, affecting children's daily lives and, consequently, their quality of life (QoL) and that of their caregivers [5].

Among the activities impaired by asthma, school attendance is frequently hampered due to asthma exacerbations, lack of asthma control, and routine medical visits, resulting in school absenteeism [6, 7]. In addition, the symptoms' burden and lack of sleep due to night wheezing impair children's at-school productivity (presenteeism), further impacting their overall school performance [8]. Moreover, children's asthma generates indirect costs, including, in addition to those associated with loss of leisure time, the ones related to their caregivers' activity and work impairment, which have been reported to be higher than direct costs [9]. Among the factors influencing school performance, previous studies have shown that good asthma control decreases school absenteeism and productivity, underscoring a role for regular asthma medication and inhaled corticosteroids $[6,10-12]$. In this context, AIT, the only treatment able to modify the course of the disease and ameliorate its symptoms, may decrease children's school and activity impairments and, consequently, those of their caregivers [13]. Among the different commercially available HDM-specific immunotherapies, Acarovac Plus ${ }^{\circledR}$ has shown a favorable effectiveness profile in previous observational studies [14-17].

Despite the previously appreciated impact of asthma on school performance and activities and, consequently, quality of life of children and adolescents, studies are insufficient and mostly focused on absenteeism [18-23]. Furthermore, to our knowledge, school and activity impairment specifically due to the most common perennial allergen, HDMs, remains unassessed. In this observational, prospective study, we assessed the impact of allergic disease on school performance and daily activities of children and adolescents with allergic asthma caused by HDMs, as well as on work and activities of their caregivers, and the effects of AIT after 1 year of treatment.

\section{Methods}

\section{Study design and population}

This was an observational, prospective and cross-sectional, multicenter study including children and adolescents with allergic asthma, with and without allergic rhinitis, and sensitized to Dermatophagoides sp. Schooled patients aged 5 to 17 years who attended visits in five Spanish allergy centers during two school years (October 2017-June 2018 and October 2018-June 2019) were consecutively included in the study. Patients had to be able to understand and complete, independently or with their accompanying adults' assistance, the administered questionnaire. Patients with concomitant diseases or circumstances potentially interfering with school attendance and patients participating or enrolled to participate in any other clinical study were excluded. According to the investigators' criteria, based on the routine clinical practice, patients with loss of school time and school impairment were prescribed AIT with Acarovac Plus® (Allergy Therapeutics, Worthing, UK). This AIT is a purified allergen extract of mites-50\% Dermatophagoides pteronyssinus $/ 50 \%$ Dermatophagoides farinae - modified with glutaraldehyde and associated to MicroCrystalline Tyrosine [MCT], in an injectable suspension for subcutaneous administration.

Data from patients and their caregivers were collected during a baseline visit (V1) and additionally, for patients who were prescribed Acarovac Plus ${ }^{\circledR}$, during a final visit (FV) 1 year after V1 (scheduled to match routine visits). Assessments were performed at V1 and FV. All legal representatives of patients signed a written informed consent before any information was recorded. The study was conducted according to the Helsinki Declaration and the local Personal Data Protection Law (LOPD 15/1999); the study protocol was approved by the Ethics Committee of "Fundació Unió Catalana Hospitals.” 


\section{Endpoints, variables, and assessments}

The primary endpoint of this study was the impact of allergic disease on school attendance and classroom performance in children and adolescents with asthma caused by Dermatophagoides spp. Secondary endpoints were the impact of allergic disease on the activity of children and adolescents with allergic asthma and on work performance and daily activities of their caregivers, and the effects of AIT in children's school and activity impairment due to allergic disease. The main variable was the impact on school impairment, measured as missed school time (i.e., absenteeism) and loss of classroom productivity (i.e., presenteeism) using the validated Spanish version of the Work Productivity and Activity Impairment Questionnaire plus Classroom Impairment Questions: Allergy Specific (WPAI + CIQ:AS), a patient-reported questionnaire validated in asthmatic patients [24-26]. The questionnaire was administered to patients during $\mathrm{V} 1$ and $\mathrm{FV}$ and to caregivers during $\mathrm{V} 1$ and included nine items assessing the time of work, school, and activities missed during the previous 7 days due to allergic disease. Specifically, the WPAI + CIQ:AS measures school and work absenteeism and presenteeism, overall school and work impairment (absenteeism plus presenteeism), and activity impairment [24]. Outcomes were calculated as the number of patients/caregivers with impairment and impairment percentages, with higher values indicating greater impairment and reduced productivity. Additional variables were essential demographic characteristics of patients and those of caregivers, such as relationship with the patient and work status. Clinical and treatment variables of patients included duration of allergic disease, sensitization to aeroallergens, other allergic diseases, number of visits to the healthcare center during the previous 12 months, use of medication to treat asthma, asthma severity classification, and asthma control. Asthma severity classification and control were graded according to the "Guía Española para el Manejo del Asma v4.0" (GEMA), the Spanish version of the Global Initiative for Asthma (GINA) classification, following, for asthma control, the classification for children $[27,28]$.

\section{Statistical analysis}

Categorical variables were described as frequencies and percentages, and quantitative variables as the mean and standard deviation (SD). Categorical variables were compared using the chi-square test and the Fisher's test. Correspondingly, quantitative variables were compared using the Student's $T$-test and their non-parametric counterparts Mann-Whitney and Kruskal-Wallis tests. The significance threshold for all bivariate analyses was set at a two-sided $\alpha=0.05$. All analyses were performed using the statistical package support SPSS.

Using the "Estimation of a proportion" method, and assuming a $22.5 \%$ loss of activities due to bronchial asthma and a $10 \%$ of data missing, a sample size of 210 patients was deemed necessary to calculate the primary endpoint (i.e., the impact of allergic disease in school attendance and performance) with a $\pm 6 \%$ precision and a $95 \% \mathrm{CI}$ [25]. Additionally, assuming a reduction of school performance due to moderate-severe allergic rhinitis of $40 \%$ and a $10 \%$ of data missing a sample size of 209 patients was deemed necessary to calculate the impact of allergic disease in school attendance and performance with $\mathrm{a} \pm 6 \%$ precision and a 95\% CI [29].

\section{Results}

\section{Baseline characteristics of study patients and accompanying adults}

Of the 125 recruited patients, 12 were unevaluable, resulting in a study population of 113 patients with a mean (SD) age of 10 (3.3) years and time of disease evolution of 4.2 (2.6) years. Table 1 summarizes the demographic, clini$\mathrm{cal}$, and treatment characteristics of study patients. The mean (SD) number of visits to healthcare centers in the previous 12 months was 3.8 (3.2). Of the 113 patients, $23 \%, 41.6 \%$, and $35.4 \%$ were recruited during the first (October-December), second (January-March), and third (April-June) quarters of the school year, respectively. The 113 accompanying caregivers had a mean (SD) age of 41.1 (6.4) years and were mostly the patients' mothers (Table 2). A flow diagram of study patients is included in Figure S1 (Supporting information file).

\section{Impairment of school/work performance and daily activities}

Allergic disease impacted the school performance and daily activities of patients, resulting in $52.2 \%$ and $45.1 \%$ prevalence of school and activity impairment, respectively (Table 3 ). Of the 113 children, $30(26.5 \%)$ reported missing at least 1 day of school, and $54(47.8 \%)$ reported classroom impairment during the previous 7 days. Likewise, working performance (evaluated in the 79 employed caregivers) and daily activities of the caregivers were impaired by children's allergic disease, with $38.0 \%$ and $23.0 \%$ prevalence of work and activity impairment, respectively (Table 3 ). 
Table 1 Demographic, clinical, and treatment characteristics of study patients, $n(\%) N=113$

\begin{tabular}{|c|c|}
\hline Demographic characteristics & \\
\hline Age, years & \\
\hline Children (5-11) & $76(67.3)$ \\
\hline Adolescents (12-17) & $37(32.7)$ \\
\hline Sex & \\
\hline Male & 75 (66.4) \\
\hline Female & 37 (33.6) \\
\hline Studies & \\
\hline Primary school & $77(68.1)$ \\
\hline Secondary school & $36(31.9)$ \\
\hline Clinical characteristics & \\
\hline Asthma severity (GEMA) & \\
\hline Intermittent & $50(44.2)$ \\
\hline Mild persistent & $28(24.8)$ \\
\hline Moderate persistent & $32(28.3)$ \\
\hline Unknown & $3(2.7)$ \\
\hline Asthma control & \\
\hline Complete & $32(28.3)$ \\
\hline Good & $51(45.1)$ \\
\hline Partial & $26(23.0)$ \\
\hline $\mathrm{Bad}$ & $1(0.9)$ \\
\hline Unknown & $3(2.7)$ \\
\hline Allergic comorbidities & \\
\hline Rhinitis & $98(87.6)$ \\
\hline Conjunctivitis & $27(23.9)$ \\
\hline Atopic dermatitis & $34(30.1)$ \\
\hline Food allergy & $9(8.0)$ \\
\hline Sensitizations to aeroallergens & \\
\hline Dermatophagoides sp. & $113(100)$ \\
\hline Other mites & $43(38.1)$ \\
\hline Pollens & $33(29.2)$ \\
\hline Alternaria alternata & $4(3.5)$ \\
\hline Treatment characteristics & \\
\hline Asthma medication & \\
\hline Short-acting beta-agonists & $62(54.9)$ \\
\hline Inhaled corticosteroids & $45(39.8)$ \\
\hline Long-acting beta-agonists & $20(17.7)$ \\
\hline Leukotriene receptor antagonists & $17(15)$ \\
\hline Oral corticosteroids & $2(1.8)$ \\
\hline
\end{tabular}

Abbreviations: GEMA, Guía Española del Manejo del Asthma (Spanish guideline on the management of asthma)

\section{Effects of immunotherapy on school performance and asthma evolution}

Of the 113 patients evaluated at V1, $65(57.5 \%)$ reported school/activity impairment and were candidates to receive AIT. Of those, 24 were not prescribed AIT based on physicians' criteria or refused AIT treatment for personal
Table 2 Demographic characteristics of caregivers, $n$ (\%) $N=113$

\begin{tabular}{ll}
\hline Sex & \\
\hline Male & $24(21.2)$ \\
Female & $86(76.1)$ \\
Unknown & $3(2.7)$ \\
Relationship & \\
Parent & $105(92.9)$ \\
Legal tutor & $1(0.9)$ \\
Grandparent & $1(0.9)$ \\
Other & $4(3.5)$ \\
Unknown & $2(1.8)$ \\
Employment status & \\
Employed & $79(69.9)$ \\
Unemployed & $27(23.9)$ \\
Retired & $2(1.8)$ \\
Other & $2(1.8)$ \\
Unknown & $3(2.7)$ \\
\hline
\end{tabular}

decision and 41 (63.1\%) were prescribed and received AIT with Acarovac Plus ${ }^{\circledR}$ (Figure S1). Twenty patients did not attend school during the week before the final visit due to the lockdown during the COVID-19 pandemic, precluding the assessment of school performance. WPAI + CIQ:AS scores of the $21(51.2 \%)$ patients who completed the first year of treatment and attended FV revealed significantly decreased absenteeism, presenteeism, overall school impairment, and activity impairment (Fig. 1). Asthma classification changed towards significantly decreased severity $(p=0.021)$ (Fig. 2A) and significantly improved control $(p=0.001)$ (Fig. 2B). Accordingly, the use of SABA and inhaled corticosteroids significantly decreased 1 year post-AIT $(p<0.05)$ (Fig. 3), and the mean (SD) number of unscheduled visits to the healthcare center decreased from 4.1 (2.8) to 1.9 (2.3) $(p=0.003)$.

\section{Discussion}

Results from this study showed a considerable prevalence of asthma-related school and activity impairment in children and adolescents allergic to HDMs and work and activity impairment in their caregivers. School and work impairment resulted from both missed school/work time and classroom/ at-work impairment, with a greater contribution of the latter. In asthmatic children receiving AIT, the severity of asthma decreased, and their control improved, with concomitantly decreased use of asthma medication, unscheduled visits to the healthcare center, and school and activity impairment 1 year after AIT.

The impact of allergic rhinitis in children's school performance and activities and that of allergic asthma in work and activity of adult patients have been evaluated in 
Table 3 Patients' school impairment, caregivers' work impairment, and patients' and caregivers' routine activities impairment due to allergic disease

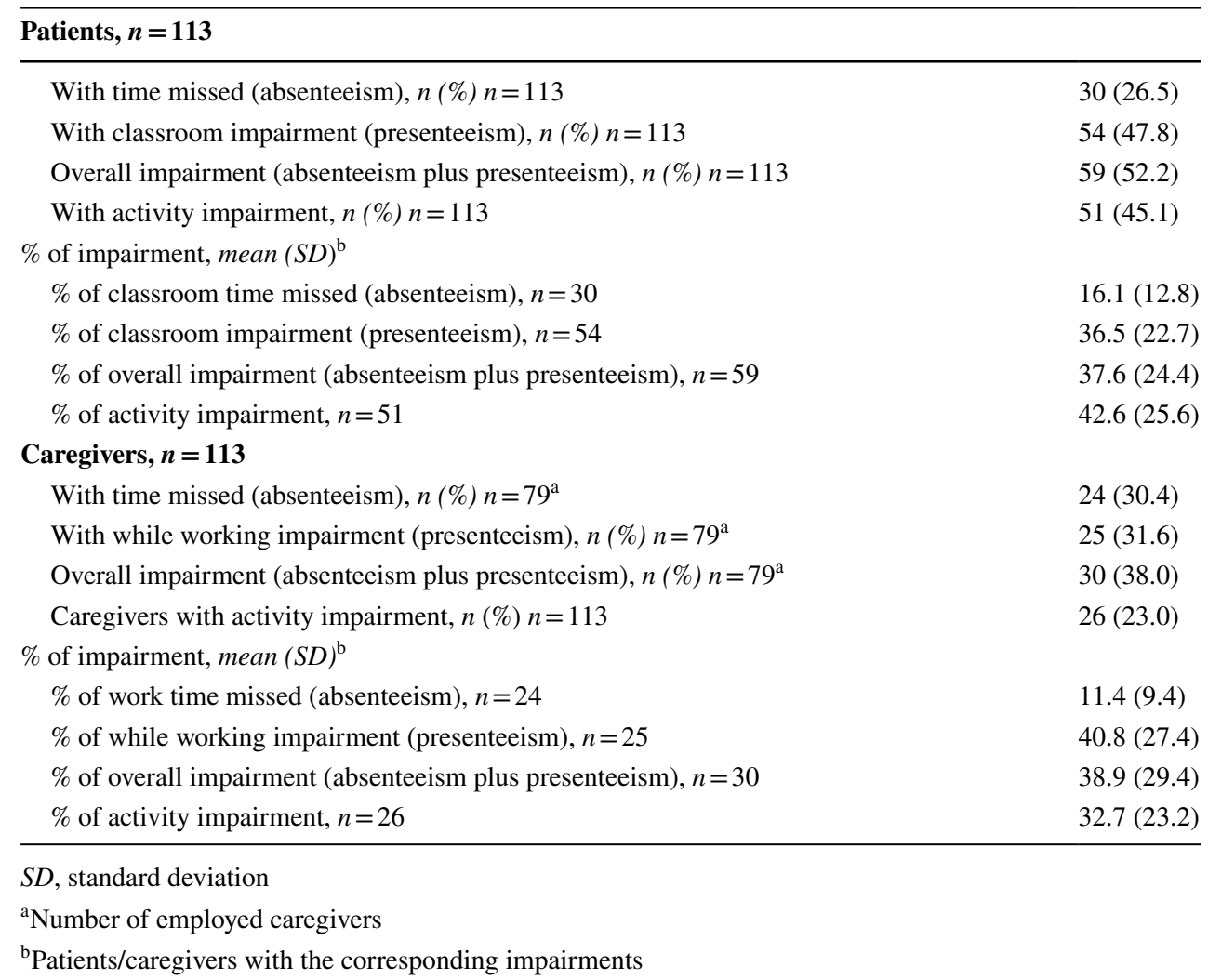

previous studies [30-32]. However, despite its persistence throughout the school year, the impact of perennial allergic asthma in children's and adolescents' daily lives and in those of their caregivers remains to be fully investigated. This study focused on the impact of perennial asthma due to allergy to HDMs, the most common allergen in children's homes during all seasons, in school performance. Using the WPAI + CIQ:AS, a standardized and widely used questionnaire specifically evaluating the impact of allergic disease, this study assessed the two dimensions of school performance: absenteeism and presenteeism [24].

The rate of school absenteeism due to asthma symptoms in this study $(26.5 \%)$ lied within the rates previously reported in different countries worldwide (ranging 9.1 to
$61 \%)$. [18, 19, 33]. Overall, this and previous studies were heterogeneous regarding time periods assessed (ranging from 7 days in this study to 12 months) and age groups, precluding direct comparisons among them. Other studies have reported increased missed school days in children with asthma compared to those without doctor-diagnosed asthma, but few of these studies tracked absences, and therefore, the direct relationship between missing school and asthma symptoms remained mostly unassessed [20, $22,34]$. Even though most of this study's population had mild persistent and intermittent asthma and good/complete asthma control, asthma symptoms had a substantial impact on the patients' school performance and daily activities. Despite differences in their design, this and previous
Fig. 1 School time missed, classroom impairment, overall impairment, and activity impairment in patients who received allergen immunotherapy with a MicroCrystalline Tyrosine-associated house dust mite allergoid and attended the final visit at the indicated timepoints. Columns and error bars represent mean percentages and standard deviation, respectively. $p$-values were calculated using the Wilcoxon test

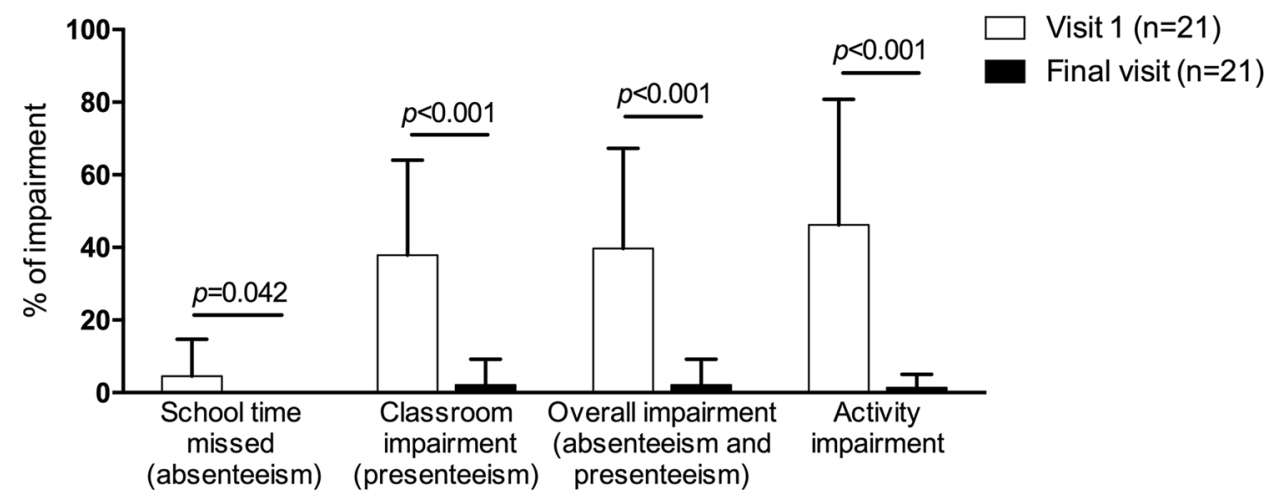


A

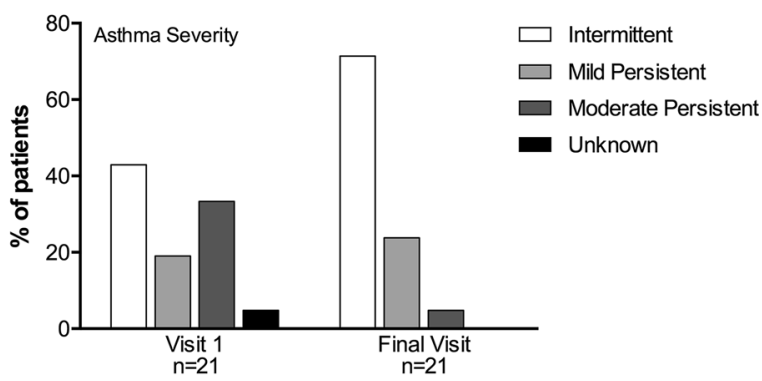

B

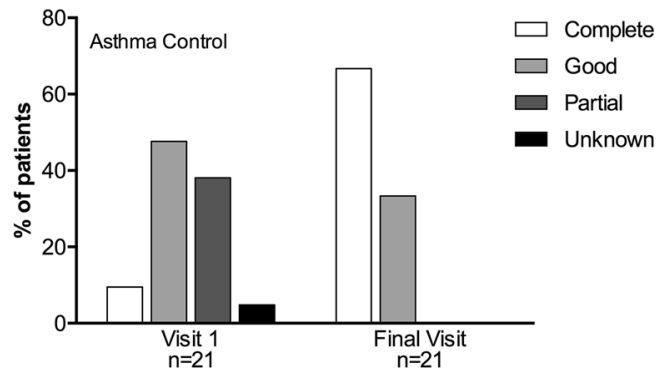

Fig. 2 Asthma severity (A) and control (B) at visit 1 and after 1 year (final visit) of treatment with allergen immunotherapy with a microcrystalline tyrosine-adsorbed house dust mite allergoid. McNemar test, $p=0.021(\mathbf{A})$ and $p=0.001(\mathbf{B})$

studies revealed a relationship between asthma and school impairment, and potentially, decreased academic achievements $[11,35]$.

Even though several studies have reported an increased probability of poor academic performance in children with asthma [11, 34, 36], few have quantified presenteeism [36]. The WPAI+CIQ:AS allowed to assess the impact of asthma in school/work presenteeism (i.e., at-school/at-work productivity loss) and showed higher rates of presenteeism (47.8\%) compared to those of absenteeism (26.5\%), underscoring a significant contribution of presenteeism to overall school impairment.

Children's asthma substantially impacts family life, including parents' sleep time and activities, and increases missing workdays, which have been shown to generate indirect costs higher than those associated with the parent's own illness [8, 9]. Likewise, the effects of asthma on work productivity have been reported to be mostly due to caregivers' presenteeism (as opposed to absenteeism), also referred to as at-work productivity [1,9]. In this context, the results from this study indicate that the impact of schooled patients' asthma on the overall work performance of their caregivers (i.e., absenteeism and presenteeism) may contribute to increasing indirect asthma costs.

Children who had received AIT to treat perennial allergic asthma due to HDM showed a favorable disease evolution, resulting in significantly decreased asthma-related school impairment in those who attended the FV. Even though

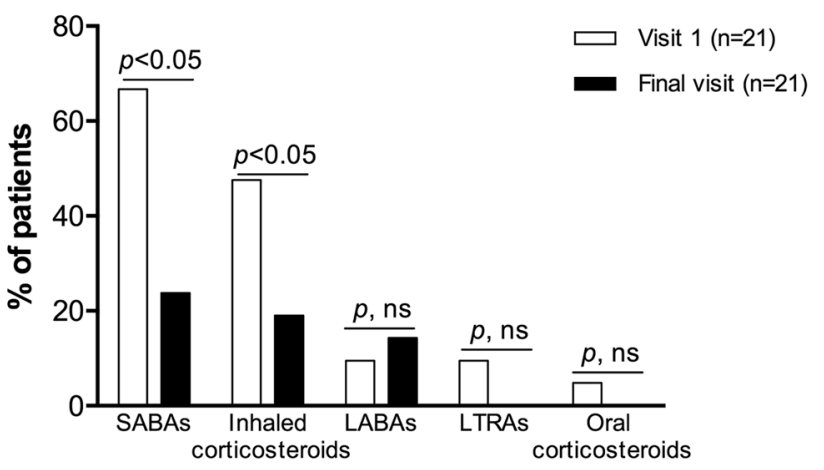

Fig. 3 Use of medication to treat asthma before and after 1 year of treatment with allergen immunotherapy with a MicroCrystalline Tyrosine-associated house dust mite allergoid. LABAs, long-acting beta-2 agonists; LTRAs, leukotriene receptor antagonists; ns, not significant; SABAs, short-acting beta- 2 agonists. $p$-values were calculated using the McNemar test

sample attrition was significant in this study, the marked reduction in school impairment was not unexpected, considering the previously reported association between the degree of asthma control and days of missed school-frequently overestimated by parents-and its severity [8, 11, 20]. In a previous study assessing the effects of this AIT in adults, work, study, and activity impairment due to rhinitis symptoms were $21.0 \%, 21.2 \%$, and $22.0 \%$, respectively, lower than the $37.6 \%$ and $42.6 \%$ for school and activity impairments, respectively, reported in this study [30]. Similar to this study, AIT was associated with increased academic and work productivity and decreased activity impairment [30]. Results from this study point to AIT with a MCT-adsorbed HDM allergoid as an effective therapeutic strategy to reduce school impairment by improving asthma evolution.

Results from this study should be interpreted in the context of limitations related to the study design and the sample used to evaluate AIT effects. Given its real-world nature, this study lacked a control arm, and consequently, changes in effectiveness variables, which were mostly subjective (i.e., symptoms and use of medication), may have been influenced by a placebo effect. However, asthma evolution was also measured using the number of visits to the healthcare center, which is a robust objective measure, and school impairment is an objective variable measured in the previous 7 days, and therefore, unlikely influenced by a potential placebo effect and recall bias. Regarding sample size, the number of patients available for analysis of the primary outcome (i.e., impact of allergic disease on school performance) did not reach the calculated study's sample size, as an insufficient number of patients was recruited. Further sample attrition for the analysis of the effects of AIT was due to the number of patients eligible to continue in the study (i.e., those with impairments) and the criteria to prescribe AIT. Finally, school lockdown during the 
COVID-19 pandemics limited the number of patients with available WPAI+CIQ:AS at 1 year of follow-up. In addition, it is possible that those patients with worse disease evolution were lost-to-follow-up, resulting in selection bias. Nevertheless, the reduced sample size provided sufficient statistical power to detect absenteeism and presenteeism and statistically significant differences after only 1 year of AIT treatment. Future studies with prolonged follow-up periods should be designed to assess the long-term effects of this AIT. Lastly, the unique characteristics of the Spanish healthcare system, free and available for everyone, facilitate access to healthcare and prescription drugs, precluding the generalizability of the results to countries with non-universal healthcare systems. Despite its limitations, this study was able to capture the impact of HDM allergic asthma in children's and their caregivers' school/work and activity impairment and the effects of AIT in the population encountered in the real-world setting, without the strict selection criteria of clinical trials. AIT resulted in favorable disease evolution and decreased school/activity impairment, and, potentially, a favorable impact on children's QoL. In addition, results from this study suggest that the indirect costs associated with loss of caregivers' work productivity due to inappropriate disease management may be reduced by therapeutic strategies able to achieve symptom control.

In conclusion, both absenteeism and presenteeism contribute to the loss of school productivity and loss of activity/leisure time due to allergic asthma symptoms in children sensitized to HDMs. AIT with a modified and MCT-associated HDM extract is likely to be effective in improving asthma control and decreasing its severity, with a concomitantly decreased school and activity impairment. Furthermore, children's asthma impacts work absenteeism, presenteeism, and daily activities of their caregivers, contributing to indirect costs of schooled children's asthma.

Supplementary Information The online version contains supplementary material available at https://doi.org/10.1007/s00431-021-04346-y.

Acknowledgements The authors would like to thank i2e3 Biomedical Research Institute for providing medical writing support during the preparation of the manuscript.

Authors' contributions Conceptualization, CG, JLJ, SN; data curation, CG, JB, VG-P, AMP, PC, JAB, ABR, LF, LF, MV, JLJ, SN; formal analysis, CG, JB, VG-P, AMP, PC, JAB, ABR, LF, LF, MV, SN, AP, CT-B, JLJ; funding acquisition, CG, SN; investigation, CG, JB, VG-P, AMP, PC, JAB, ABR, LF, LF, MV, JLJ, SN; methodology, CG, JB, VG-P, AMP, PC, JAB, ABR, LF, LF, MV, JLJ, SN; project administration, CG, SN, CT-B, AP, JLJ; resources, CG, JB, VG-P, AMP, PC, JAB, ABR, LF, LF, MV, SN; software, CG, JB, VG-P, AMP, PC, JAB, ABR, LF, LF, MV, JLJ, SN; supervision, CG, SN, CT-B, AP, JLJ; validation, CG, JB, VG-P, AMP, PC, JAB, ABR, LF, LF, MV, JLJ, SN; visualization, CG, CT-B, JLJ; writing - original draft preparation, CG, CT-B; writing - review and editing, CG, JB, VG, AMP, PC, JAB, ABR, LF, LF, MV, SN, CT-B, AP, JLJ.
Funding This study was supported by Allergy Therapeutics Ibérica (Sant Joan Despí, Barcelona, Spain).

Availability of data and material The datasets used and/or analyzed during the current study are available from the corresponding author upon reasonable request.

Code availability Not applicable.

\section{Declarations}

Ethics approval The study was conducted according to the Helsinki Declaration and the local Personal Data Protection Law (LOPD 15/1999); the study protocol was approved by the Ethics Committee of "Fundació Unió Catalana Hospitals."

Consent to participate All legal representatives of patients signed a written informed consent before any information was recorded.

Consent for publication Not applicable.

Conflict of interest CB, JB, VG-P, AMP, PC, JAB, ABR, LF, LF, MV, and SN declare that they have no conflict of interests. CT-B, AP, and JLJ are employees of Allergy Therapeutics.

Open Access This article is licensed under a Creative Commons Attribution 4.0 International License, which permits use, sharing, adaptation, distribution and reproduction in any medium or format, as long as you give appropriate credit to the original author(s) and the source, provide a link to the Creative Commons licence, and indicate if changes were made. The images or other third party material in this article are included in the article's Creative Commons licence, unless indicated otherwise in a credit line to the material. If material is not included in the article's Creative Commons licence and your intended use is not permitted by statutory regulation or exceeds the permitted use, you will need to obtain permission directly from the copyright holder. To view a copy of this licence, visit http://creativecommons.org/licenses/by/4.0/.

\section{References}

1. Dierick BJH, van der Molen T, Flokstra-de Blok BMJ et al (2020) Burden and socioeconomics of asthma, allergic rhinitis, atopic dermatitis and food allergy. Expert Rev Pharmacoecon Outcomes Res 20:437-453. https://doi.org/10.1080/14737167.2020.1819793

2. Pearce N, Aït-Khaled N, Beasley R et al (2007) Worldwide trends in the prevalence of asthma symptoms: phase III of the International Study of Asthma and Allergies in Childhood (ISAAC). Thorax 62:758-766. https://doi.org/10.1136/thx.2006.070169

3. Agache I, Lau S, Akdis CA et al (2019) EAACI guidelines on allergen immunotherapy: house dust mite-driven allergic asthma. Allergy 74:855-873. https://doi.org/10.1111/all.13749

4. Calderón MA, Linneberg A, Kleine-Tebbe J et al (2015) Respiratory allergy caused by house dust mites: what do we really know? J Allergy Clin Immunol 136:38-48. https://doi.org/10.1016/j.jaci. 2014.10.012

5. Asher MI, Montefort S, Björkstén B et al (2006) Worldwide time trends in the prevalence of symptoms of asthma, allergic rhinoconjunctivitis, and eczema in childhood: ISAAC phases one and three repeat multicountry cross-sectional surveys. Lancet (London, England) 368:733-743. https://doi.org/10.1016/S01406736(06)69283-0 
6. Schmier JK, Manjunath R, Halpern MT et al (2007) The impact of inadequately controlled asthma in urban children on quality of life and productivity. Ann Allergy Asthma Immunol 98:245-251. https://doi.org/10.1016/S1081-1206(10)60713-2

7. Prevention C for DC and CDC Healthy Schools. Asthma

8. Carroll WD, Wildhaber J, Brand PLP (2012) Parent misperception of control in childhood/adolescent asthma: the room to breathe survey. Eur Respir J 39:90-96. https://doi.org/10.1183/09031936. 00048911

9. Stróżek J, Samoliński B, Kłak A et al (2019) The indirect costs of allergic diseases. Int J Occup Med Environ Health 32:281-290. https://doi.org/10.13075/ijomeh.1896.01275

10. Toyran M, Yagmur IT, Guvenir H et al (2020) Asthma control affects school absence, achievement and quality of school life: a multicenter study. Allergol Immunopathol (Madr) 48:545-552. https://doi.org/10.1016/j.aller.2020.05.005

11. Moonie S, Sterling DA, Figgs LW, Castro M (2008) The relationship between school absence, academic performance, and asthma status. J Sch Health 78:140-148. https://doi.org/10.1111/j.17461561.2007.00276.x

12. Tsakiris A, Iordanidou M, Paraskakis E et al (2013) The presence of asthma, the use of inhaled steroids, and parental education level affect school performance in children. Biomed Res Int 2013:762805. https://doi.org/10.1155/2013/762805

13. Bousquet J, Lockey R, Malling H (1998) Allergen immunotherapy: therapeutic vaccines for allergic diseases A WHO position paper. J Allergy Clin Immunol 102:558-562. https://doi.org/10. 1016/S0091-6749(98)70271-4

14. Roger A, Depreux N, Jurgens Y et al (2014) A novel and well tolerated mite allergoid subcutaneous immunotherapy: evidence of clinical and immunologic efficacy. Immunity, Inflamm Dis 2:92-98. https://doi.org/10.1002/iid3.23

15. Roger A, Malet A, Moreno V et al (2020) Real-life effect of a microcrystalline tyrosine adjuvanted mite immunotherapy in patients with allergic rhinitis. Immunotherapy 12:53-62. https:// doi.org/10.2217/imt-2019-0205

16. Sala-Cunill A, Pérez-Formoso JL, Torán-Barona C et al (2020) Safety and effectiveness of a microcrystalline tyrosine-associated mite extract immunotherapy for allergic rhinitis. Immunotherapy 12:1007-1019. https://doi.org/10.2217/imt-2020-0194

17. Sala-Cunill A, Almeida-Sánchez ZM, García-Núñez I et al (2021) Real-world safety and effectiveness evidence of a microcrystalline tyrosine-associated mite allergoid in children and adolescents with allergic rhinitis. Allergol Immunopathol (Madr) 49:98-108. https://doi.org/10.15586/aei.v49i4.195

18. Hsu J, Qin X, Beavers SF, Mirabelli MC (2016) Asthma-related school absenteeism, morbidity, and modifiable factors. Am J Prev Med 51:23-32. https://doi.org/10.1016/j.amepre.2015.12.012

19. Kim S-H, Sohn K-H, Kang S-Y et al (2018) School absenteeism associated with asthma and allergic diseases in Korean schoolaged children. Pediatr Allergy Immunol Pulmonol 31:151-157. https://doi.org/10.1089/ped.2017.0835

20. Moonie SA, Sterling DA, Figgs L, Castro M (2006) Asthma status and severity affects missed school days. J Sch Health 76:18-24. https://doi.org/10.1111/j.1746-1561.2006.00062.x

21. Freudenberg N, Feldman CH, Clark NM et al (1980) The impact of bronchial asthma on school attendance and performance. J Sch Health 50:522-526. https://doi.org/10.1111/j.1746-1561.1980.tb02079.x
22. Silverstein MD, Mair JE, Katusic SK et al (2001) School attendance and school performance: a population-based study of children with asthma. J Pediatr 139:278-283. https://doi.org/10.1067/ mpd.2001.115573

23. Vuurman E, van Veggel L, Uiterwijk M et al (1993) Seasonal allergic rhinitis and antihistamine effects on children's learning. Ann Allergy 71:121-126

24. Reilly MC, Zbrozek AS, Dukes EM (1993) The validity and reproducibility of a work productivity and activity impairment instrument. Pharmacoeconomics 4:353-365. https://doi.org/10.2165/ 00019053-199304050-00006

25. Andréasson E, Svensson K, Berggren F (2003) Validity of the work productivity and activity impairment questionnaire for patients with asthma (WPAI-asthma): results from a web-based study. In: ISPOR Congress 2003

26. Associates R No Title. In: WPAI Transl http://www.reillyassociates. net/WPAI_Translations.html. Accessed 17 Jan 2021

27. Comité Ejecutivo de la GEMA.(2015) GEMA 4.0. Guía Española para el Manejo del Asma. http://www.semg.es/images/stories/ recursos/2015/documentos/GEMA_4.0_2015.pdf

28. Global Initiative for Asthma (GINA) (2019) Global strategy for asthma management and prevention

29. Bousquet J, Neukirch F, Bousquet PJ et al (2006) Severity and impairment of allergic rhinitis in patients consulting in primary care. J Allergy Clin Immunol 117:158-162. https://doi.org/10. 1016/j.jaci.2005.09.047

30. Roger A, Arcalá Campillo E, Torres MC et al (2016) Reduced work/academic performance and quality of life in patients with allergic rhinitis and impact of allergen immunotherapy. Allergy, Asthma Clin Immunol 12:40. https://doi.org/10.1186/ s13223-016-0146-9

31. Devillier P, Bousquet J, Salvator $\mathrm{H}$ et al (2016) In allergic rhinitis, work, classroom and activity impairments are weakly related to other outcome measures. Clin Exp Allergy 46:1456-1464. https:// doi.org/10.1111/cea.12801

32. Lee LK, Ramakrishnan K, Safioti G et al (2020) Asthma control is associated with economic outcomes, work productivity and health-related quality of life in patients with asthma. BMJ open Respir Res 7. https://doi.org/10.1136/bmjresp-2019-000534

33. Centers for Disease Control and Prevention. National Asthma Control Program (2013) Asthma statistics: asthma related missed school days among children aged 5-17 years

34. Mizan SS, Shendell DG, Rhoads GG (2011) Absence, extended absence, and repeat tardiness related to asthma status among elementary school children. J Asthma 48:228-234. https://doi.org/ 10.3109/02770903.2011.555038

35. Schneider T (2020) Asthma and academic performance among children and youth in North America: a systematic review. J Sch Health 90:319-342. https://doi.org/10.1111/josh.12877

36. Basch CE (2011) Asthma and the achievement gap among urban minority youth. J Sch Health 81:606-613. https://doi.org/10. 1111/j.1746-1561.2011.00634.x

Publisher's Note Springer Nature remains neutral with regard to jurisdictional claims in published maps and institutional affiliations. 